\title{
Totally Laparoscopic D2 Subtotal Gastrectomy with Hepatectomy and Cholecystectomy for Gastric Neuroendocrine Tumor with Liver Metastases
}

\author{
Brian K. P. Goh, MBBS, MMed, MSc, FRCS, Alvin K. H. Eng, MBBS, MMed, MSc, FRCS \\ Department of Surgery, Singapore General Hospital, Singapore (all authors). \\ Duke-NUS Graduate Medical School, Singapore (Dr. Goh).
}

\begin{abstract}
Synchronous resection of primary neuroendocrine tumors (NET) with liver metastases remains controversial, although recent studies have demonstrated its safety in select patients. Synchronous laparoscopic gastric and liver resection has been rarely reported. We report the case of a 65-year-old man who underwent successful simultaneous D2 subtotal gastrectomy with hepatectomy and cholecystectomy for metastatic gastric NET. This is the first reported case of totally laparoscopic simultaneous D2 gastrectomy with hepatectomy and cholecystectomy for metastatic gastric NET. Laparoscopic simultaneous gastrectomy and hepatectomy is feasible and safe in select patients with metastatic gastric NET.
\end{abstract}

Key Words: Laparoscopy, Hepatectomy, Gastrectomy, Simultaneous, Neuroendocrine tumor, liver, gastric, resection, carcinoid.

Citation Goh BKP, Eng AKH. Totally laparoscopic D2 subtotal gastrectomy with hepatectomy and cholecystectomy for gastric neuroendocrine tumor with liver metastases. CRSLS e2014.00025. DOI: 10.4293/CRSLS.2014.00025.

Copyright (C) 2014 SLS This is an open-access article distributed under the terms of the Creative Commons Attribution-Noncommercial-ShareAlike 3.0 Unported license, which permits unrestricted noncommercial use, distribution, and reproduction in any medium, provided the original author and source are credited.

Address correspondence to: Brian K. P. Goh, MBBS, MMed, MSc, FRCS, Consultant, Hepatopancreatobiliary and Transplantation Service, Department of Surgery, Singapore General Hospital, Outram Road, Singapore 169608. E-mail: bsgkp@ hotmail.com. Phone: 65-63214051

\section{INTRODUCTION}

Neuroendocrine tumors (NETs) are rare, with a reported incidence of 4 to 5 cases per 100,000 population per year in the United States. ${ }^{1}$ They are usually slow growing with a favorable prognosis. ${ }^{2}$ However, liver metastases are a common occurrence and are reported to occur synchronously with the primary tumor in $50 \%$ of patients. ${ }^{3}$ The optimal treatment of liver metastases is presently unknown. ${ }^{4}$ Accepted treatment modalities include surgical resection and nonsurgical modalities such as local ablative therapy, intraarterial locoregional therapy, chemotherapy, and radiotherapy. Nonetheless, most investigators currently would recommend aggressive surgical resection if an R0 resection can be achieved in well-differentiated NETs. ${ }^{2}$ Synchronous resection of both the primary NET and liver metastases remains controversial, although a recent study from Memorial-Sloan Kettering Cancer Center demonstrated its safety in 36 highly selected patients with primary or recurrent NET. ${ }^{2}$

When laparoscopic surgery was first introduced for abdominal malignancies, it was initially met with much skepticism. ${ }^{5}$ However, currently, laparoscopic surgery has been demonstrated to be safe, with similar oncologic outcomes to open surgery for various abdominal malignancies such as colon, liver, and gastric cancers. ${ }^{6-8}$ Nonetheless, despite the increasing use of laparoscopic resection for abdominal malignancies, totally laparoscopic simultaneous resection of metastatic carcinomas remains rare because of the complexity of these procedures.5,9 Combined colon and liver resection for stage IV colorectal cancer is currently probably the most common indication for simultaneous resections for metastatic carcinoma. In a recent retrospective analysis of the United States Nationwide Inpatient Sample conducted from 2002 to 2006, 3625 such cases were performed. ${ }^{10}$ This database represents $\sim 20 \%$ of the stratified sample of US community hospitals. ${ }^{10}$ Nevertheless, simultaneous totally laparoscopic colon and hepatic resection has only been rarely reported. 5,9

Currently, simultaneous combined gastric and liver resection is extremely rare because complete surgical resection of liver metastases from metastatic gastric carcinoma is extremely controversial and not well accepted. ${ }^{11}$ On one 
hand, simultaneous resection of metastatic gastric NET to the liver is more acceptable and has been reported, albeit rarely, worldwide. ${ }^{2,12}$ In this study, we present to our knowledge the first reported case in the English literature of totally laparoscopic simultaneous D2 gastrectomy with hepatectomy and cholecystectomy for metastatic gastric NET.

\section{CASE REPORT}

A 65-year-old man was admitted to the hospital with stable upper gastrointestinal bleeding. Gastroscopy revealed a $3-\mathrm{cm}$ tumor arising from the antrum, requiring a biopsy. Histologic examination confirmed a grade 2 NET. Staging computed tomographic (CT) scan demonstrated the $3-\mathrm{cm}$ polypoidal tumor within the stomach with a $2.5-\mathrm{cm}$ mass in segment $4 \mathrm{~b}$ of the liver (Figures 1 and $\mathbf{2}$ ). Serum chromogranin A was elevated at $298.5 \mu \mathrm{g} / \mathrm{L}$ (normal range, $0-100 \mu \mathrm{g} / \mathrm{L}$ ). The rest of the biochemical tests, including serum fasting gastrin, liver function, and 24hour urine 5-OH indole acetic acid (5-HIAA), demonstrated results within normal limits. The patient was diagnosed with type 3 gastric carcinoid with liver metastases. A staging 68Ga-DOTATATE positron emission tomography scan (Figure 3) was performed and showed the primary carcinoid tumor in the stomach with regional lymph node involvement (gastrohepatic ligament) and 3 metastatic nodules in the liver ( 1 corresponding to the

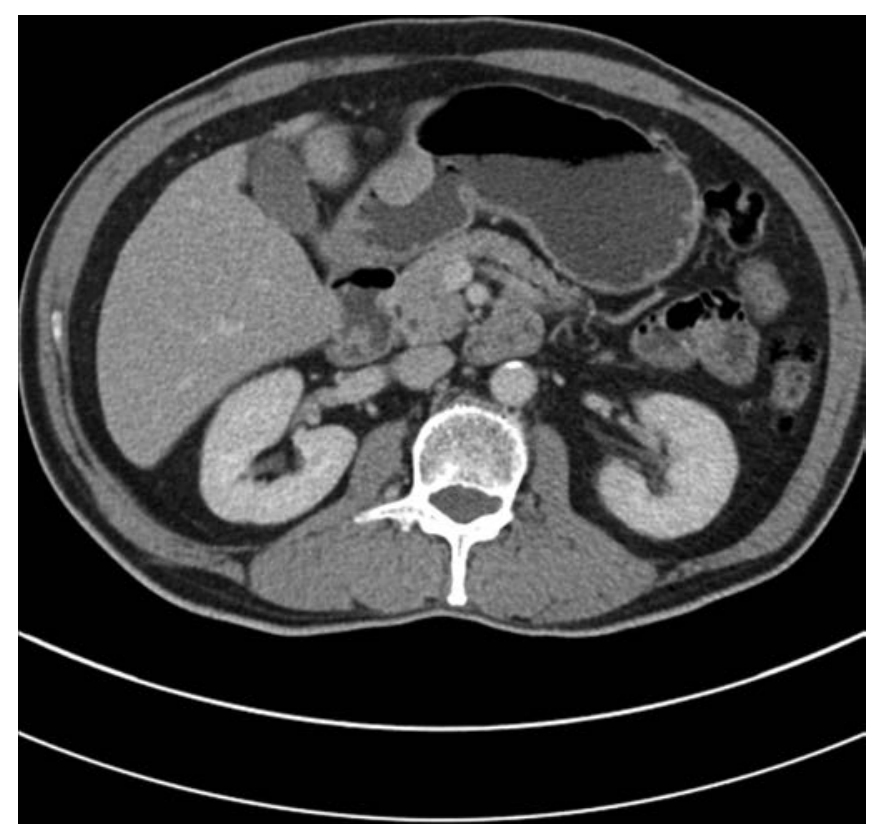

Figure 1. A CT scan demonstrating the $3-\mathrm{cm}$ polypoidal tumor rising from the gastric antrum.

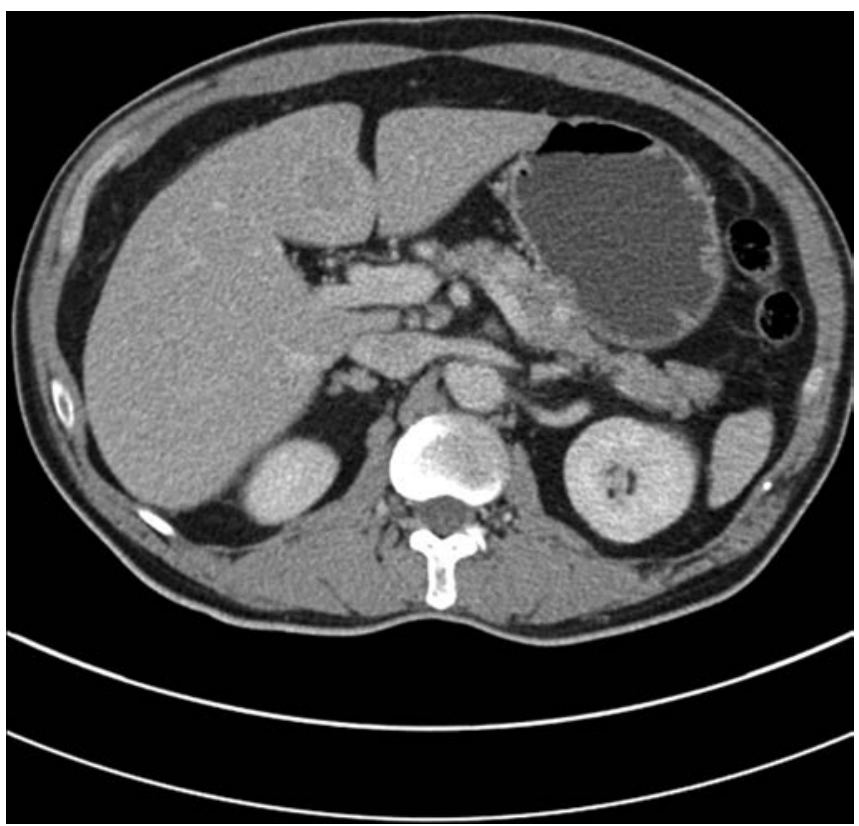

Figure 2. A $2.5-\mathrm{cm}$ lesion in segment IVb.

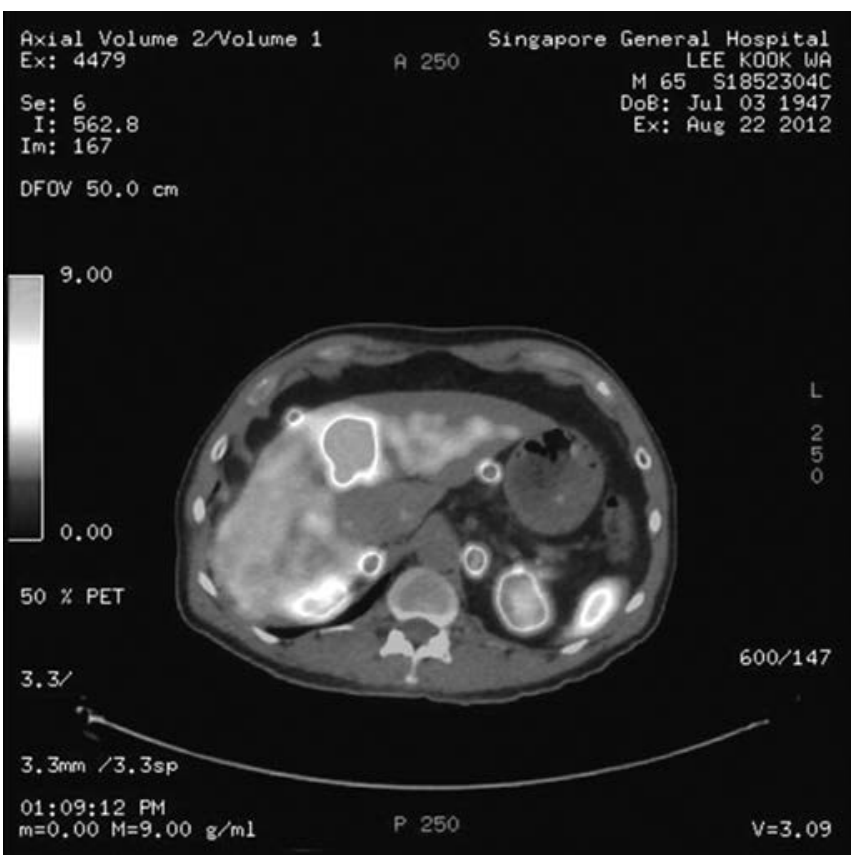

Figure 3. A 68Ga-DOTATATE positron emission tomography scan demonstrating the $2.5-\mathrm{cm}$ segment IVb metastatic nodule, with an adjacent sub-centimeter nodule on the surface of the right lobe. There was also regional lymph node involvement within the gastrohepatic ligament. 
lesion in segment IVb on CT and 2 lesions on the surface of the right lobe). There were no other extrahepatic metastases. A laparoscopic combined D2-subtotal gastrectomy and resection of the liver metastases with cholecystectomy was planned for the patient.

For surgery, the patient was placed in the reverse Trendelenburg position with his legs apart, and the surgeon stood between his legs, with the cameraman and assistant to the left. The $12-\mathrm{mm}$ umbilical port was created via open technique (Figure 4A). After a pneumoperitoneum was established, 4 additional ports were inserted (two 12-mm ports and two 5-mm ports (Figure 4B-E). Intraoperative ultrasonography confirmed the 3 nodules in the liver. Cholecystectomy, segment IVb resection, and metastectomy of the two superficial $5-\mathrm{mm}$ nodules were performed. Hepatic parenchymal resection of segment $\mathrm{IVb}$ was performed using the combination of a Cavitron Ultrasonic Surgical Aspirator (CUSA) (Valleylab, Boulder, CO)

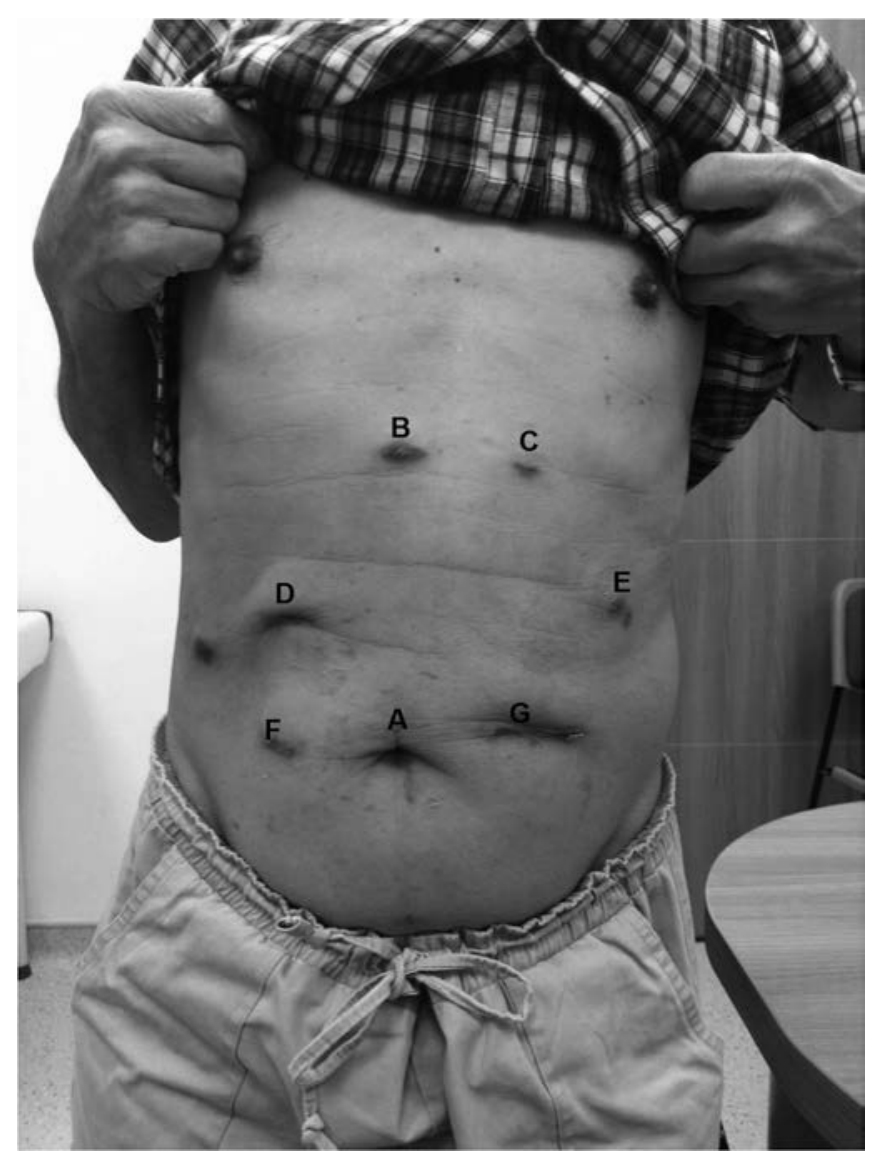

Figure 4. Incisions and port placement on the patient's abdomen. A, umbelical port; B to E, ports per hepatectomy; F to G, additional ports for gastrectomy. and an Enseal device (Ethicon Endo-Surgery, Cincinnati, $\mathrm{OH})$. Larger hepatic pedicles were clipped and divided. The Harmonic scalpel (Ethicon Endo-Surgery) was used to resect the two superficial nodules on the surface of the right lobe. The resected specimens were placed in a plastic retrieval bag and left in the peritoneal cavity.

The patient was repositioned into the supine position for the gastrectomy and two 12-mm ports were inserted (Figure $4 \mathbf{F}$ and $\mathbf{G})$. The surgeon and cameraman were on the patient's right side. The liver was retracted with a liver retractor, and dissection was performed using the Harmonic scalpel (Ethicon Endo-Surgery). The gastrocolic ligament was divided, completing $\# 4 \mathrm{SB}$ and $\# 4 \mathrm{D}$, and the right gastroepipolic artery and vein were doubly clipped and divided. The infrapyloric vessels were clipped and divided, taking \#6 nodes. Subsequently, supraduodenal dissection was performed along the gastroduodenal artery, common hepatic artery, and proper hepatic artery, completing the $\# 5, \# 12 \mathrm{~A}, \# 8$, and $\# 8 \mathrm{P}$ lymph node dissections. The right gastric artery was divided between clips. The duodenum was divided using the motorized Echelon Flex 60 with blue cartridge (Ethicon Endo-Surgery). The left gastric artery and vein were clipped and divided, completing \#7 and \#9 lymphadenectomy. Dissection along the splenic artery was performed to complete \#11P and, finally, $\# 1$ and $\# 3$ dissections were completed before a proximal gastric transection was performed using the motorized stapler with blue cartridge (two fires). The left port side (Figure $\mathbf{4 G}$ ) was enlarged to $4 \mathrm{~cm}$ transversely, and the gastric and liver specimens were retrieved. Intracorporeal Bilroth II reconstruction was performed with the stapler, and the enterotomies were sutured closed in a continuous fashion. Tisseel glue (Baxter, Chicago, IL) and Surgicel (Ethicon Biosurgery, Somerville, NJ) were applied on the raw liver surface. Finally, two closed suction drains were placed with the tips into Morrison's pouch and to the left subphrenic space.

The total operative time was 575 minutes and the estimated blood loss was $250 \mathrm{~mL}$. No intraoperative blood transfusion was required. The patient's postoperative recovery was uneventful and he was discharged on postoperative day 6 . Final histologic results demonstrated a $3-\mathrm{cm}$ well-differentiated NET of the stomach, with 2 of 49 lymph nodes positive for metastases. The mitotic count was 6 per $10 \mathrm{hpf}$. The 3 resected liver nodules were also confirmed to be NET metastases with clear margins. The patient was readmitted on postoperative day 10 with symptoms suggestive of delayed gastric emptying. Gastroscopy demonstrated that both efferent and afferent limbs were widely patent, and a contrast study showed delayed but free flow 
of contrast down the efferent limb. The patient was gradually progressed to an oral diet and was discharged well on postoperative day 18 . He remained symptom-free on review at postoperative day 32 .

\section{DISCUSSION}

The optimal treatment strategy for metastatic NET is presently not well defined. Synchronous resection for metastatic NETs has not been well addressed, although a recent study reported the safety of this approach in 36 patients who underwent surgery at the Memorial Sloan-Kettering Cancer Center. ${ }^{2}$ The overall morbidity was $44 \%$, and there was one case of mortality after a Whipple procedure with right trisectionectomy. ${ }^{2}$ The 5 -year overall survival was $69 \%$. In the series, 3 patients had a gastrectomy for the primary tumor, with concomitant liver resection. The possible advantages of synchronous resection include (1) avoidance of the complications of the primary tumor including bleeding and obstruction, (2) improved survival, (3) improved long-term symptom control, and (4) reduced total morbidity and length of stay by avoiding a second surgery. ${ }^{2}$ Routine cholecystectomy should be performed during synchronous resection to avoid the potential complications associated with the future use of hepatic arterial embolizations and somatostatin analogs. ${ }^{2}$

In the present study, we have described the first case of totally laparoscopic simultaneous D2 subtotal gastrectomy with hepatectomy and cholecystectomy for metastatic gastric NET. Although laparoscopic D2 gastrectomy is routinely performed in many Asian countries for gastric carcinoma, ${ }^{8}$ simultaneous laparoscopic gastric and hepatic resection has never been reported because hepatic resection is almost never indicated for liver metastases from gastric carcinoma. However, this combined approach should be considered in carefully selected patients who may benefit from the various advantages offered by minimally invasive surgery. The obvious limitation of this combined procedure lies in its complexity, as evidenced by the long operating time. Nonetheless, we have demonstrated its safety as shown by the minimal blood loss and the absence of major postoperative morbidity. When complex laparoscopic surgery is undertaken, it is important to remember that conversion is considered prudent surgical practice rather than failure. It should be performed when patient safety is potentially compromised, such as when there is significant blood loss or there is failure to progress, resulting in extended operating times. ${ }^{7}$

A major determinant in the selection of patients for this complex simultaneous laparoscopic procedure lies in the experience and technical ability of the operating surgeon. For laparoscopic D2 gastrectomy, size and location of the tumor are two major factors that determine the complexity of the procedure. Larger tumors are frequently difficult to manipulate laparoscopically, whereas proximally located tumors may require a total gastrectomy. However, these are not absolute contraindications to the procedure because laparoscopic total gastrectomy ${ }^{12}$ and laparoscopic gastrectomy for advanced stage gastric cancers ${ }^{13}$ are routinely performed in certain highly specialized centers. This is similar for laparoscopic hepatectomy, whereby tumor size and location are the two important determinants of the complexity of the surgery. Tumors located in the "accessible" segments, such as segments 2, 3, 4b, 5, and 6, are the most amenable to laparoscopic resection. ${ }^{8}$ However, this is again dependent on the operating surgeon's ability and experience; expert surgeons have reported successful laparoscopic resection of all segments in the liver including the right posterior segments (segments 7 and 8), caudate lobe, and even living donor right hepatectomies. ${ }^{14,15}$

In an effort to reduce the operating time and complexity of certain laparoscopic procedures, various minimally invasive strategies have been used as a complement or an alternative to totally laparoscopic surgery, including handassisted laparoscopic and laparoscopic-assisted surgery. These techniques may be adopted by less experienced surgeons, especially during the early phase of the learning curve. As in laparoscopic colectomy, laparoscopic-assisted gastrectomy, whereby the anastomosis is performed extracorporeally, is more widely performed than totally laparoscopic gastrectomy. This is likely because of the significant time savings afforded by the latter approach. The hand-assisted laparoscopic approach to gastrectomy may also result in a shorter operating time compared with the purely laparoscopic approach. ${ }^{16}$ For similar reasons, the laparoscopic-assisted and hand-assisted approaches have been proposed for hepatectomies, especially for major hepatectomies and tumors located in positions that are difficult to access. ${ }^{17}$ At this time, it is uncertain which of these techniques is superior.

In conclusion, laparoscopic simultaneous D2 gastrectomy with hepatectomy and cholecystectomy is feasible and safe and should be considered in select patients with metastatic gastric NET.

\section{References:}

1. Hauso O, Gustafsson BI, Kidd M, et al. Neuroendocrine tumor epidemiology: contrasting Norway and North America. Cancer. 2008;113:2655-2664. 
2. Gaujoux S, Gonen M, Tang L, et al. Synchronous resection of primary and liver metastases for neuroendocrine tumors. Ann Surg Oncol. 2012;19:4270-4277.

3. Modlin IM, Sandor A. An analysis of 8305 cases of carcinoid tumors. Cancer. 1997;79:813-829.

4. Gurusamy KS, Ramamoorthy R, Sharma D, Davidson RR. Liver resection versus other treatments for neuroendocrine tumours in patients with resectable liver metastases. Cochrane Database Syst Rev. 2009;(1):CD007060.

5. Casaccia M, Famiglietti F, Andorno E, Domenico SD, Ferrari C, Valente U. Simultaneous laparoscopic anterior resection and left hepatic lobectomy for stage IV rectal cancer. JSLS. 2010;14: $414-417$.

6. Kuhry E, Schwenk W, Gaupset R, Romild U, Bonier J. Longterm outcome of laparoscopic surgery for colorectal cancer: a Cochrane systematic review of randomised controlled trials. Cancer Treat Rev. 2008;34:498-504.

7. Buell JF, Cherqui D, Geller DA, et al. The international position paper on laparoscopic liver surgery: the Louisville statement, 2008. Ann Surg. 2009;250:825-830.

8. Liang Y, Li G, Chen P, Yu J, Zhang C. Laparoscopic versus open gastrectomy for early distal gastric cancer: a meta-analysis. ANZ J Surg. 2011;81:673-680.

9. Lupinacci RM, Machado MA, Lupinacci RA, Herman P. Simultaneous left colectomy and standard hepatectomy performed by laparoscopy. Rev Col Bras Cir. 2011;38(2):139-141.

10. Yang XW, Li Z, Liu K, Fu XH, Yang JH, Wu MC. Correlation between the survival rate of patients with synchronous hepatic metastases from gastric carcinoma after surgical resection and patient's index. Chin Med J (Engl). 2012;125(5):747-751.

11. Elias D, Lasser P, Ducreux M, et al. Liver resection (and associated extrahepatic resections) for metastatic well-differentiated endocrine tumors: a 15-year single center prospective study. Surgery. 2003;133:375-382.

12. Kim HS, Kim BS, Lee IS, Lee S, Yook JH, Kim BS. Comparison of totally laparoscopic total gastrectomy and open total gastrectomy for gastric cancer. J Laparoendosc Adv Surg Tech A. 2013;23:323-331.

13. Lee JH, Son SY, Lee CM, Ahn SH, Park DJ, Kim HH. Morbidity and mortality after laparoscopic gastrectomy for advanced gastric cancer: results of a phase II clinical trial. Surg Endosc. 2013 Feb 13 [Epub ahead of print].

14. Yoon YS, Han HS, Cho JY, Ahn KS. Total laparoscopic liver resection for hepatocellular carcinoma located in all segments of the liver. Surg Endosc. 2010;24:1630-1637.

15. Ishizawa T, Gumbs AA, Kokudo N, Gayet B. Laparoscopic segmentectomy of the liver: from segment I to VIII. Ann Surg. 2012;256:959-964.

16. Wong SK, Tsui DK, Li MK. Laparoscopic distal gastrectomy for gastric cancer: initial experience on hand-assisted technique and totally laparoscopic technique. Surg Laparosc Endosc Percutan Tech. 2009;19:298-304.

17. Lin NC, Niita H, Wakabayashi G. Laparoscopic major hepatectomy: a systematic literature review and comparison of 3 techniques. Ann Surg. 2013;257:205-213. 